\title{
Evaluation of Fast-Time Wake Models using Denver 2006 Field Experiment Data
}

\author{
Nash'at N. Ahmad* \\ NASA Langley Research Center, Hampton, Virginia, 23681 \\ Matthew J. Pruis ${ }^{\dagger}$ \\ NorthWest Research Associates, Redmond, Washington, 98052
}

The National Aeronautics and Space Administration conducted a series of wake vortex field experiments at Denver in 2003, 2005, and 2006. This paper describes the lidar wake vortex measurements and associated meteorological data collected during the 2006 deployment, and includes results of recent reprocessing of the lidar data using a new wake vortex algorithm and estimates of the atmospheric turbulence using a new algorithm to estimate eddy dissipation rate from the lidar data. The configuration and set-up of the 2006 field experiment allowed out-of-ground effect vortices to be tracked in lateral transport further than any previous campaign and thereby provides an opportunity to study long-lived wake vortices in moderate to low crosswinds. An evaluation of NASA's fast-time wake vortex transport and decay models using the dataset shows similar performance as previous studies using other field data.

\section{Nomenclature}

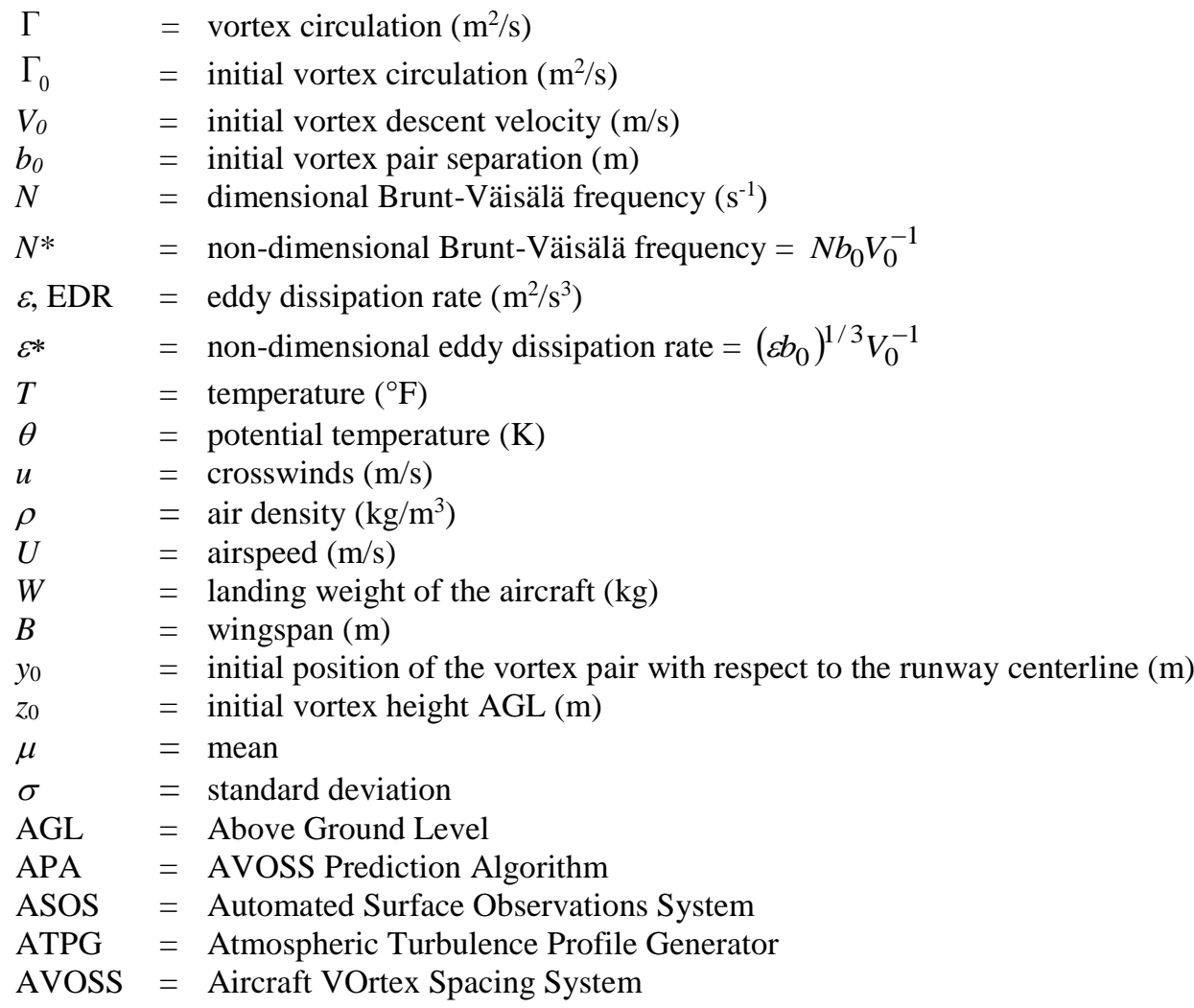

\footnotetext{
${ }^{*}$ Research Aerospace Engineer, NASA, Hampton, Virginia. Senior Member, AIAA.

${ }^{\dagger}$ Research Scientist, NWRA, Redmond, Washington. Member, AIAA.
} 
OGE $\quad=$ Out of Ground Effect

TASS $=$ Terminal Area Simulation System

TDP $=$ TASS Derived Algorithms for Wake Prediction

WakeMod $=$ improved algorithm for estimating circulation of aircraft wake vortices (Version 2.5)

\section{Introduction}

$\mathrm{O}$ VER the years, the National Aeronautics and Space Administration has conducted a series of wind tunnel tests (Chow et al. 1997), flight tests (Vicroy et al. 1998) and several field experiments to characterize wake vortices and to study the effect of ambient atmospheric conditions on their decay and transport. In addition to providing important insight into vortex physics, the data from these experiments and flight tests have been used to develop and evaluate fast-time wake vortex prediction models. Building upon earlier studies, NASA performed a large field experiment at Memphis International Airport in 1995 to measure wake vortex decay and transport characteristics under varying atmospheric conditions and for many different types of aircraft (Campbell, et al. 1997). The experiment was sponsored under NASA Langley Research Center's Aircraft Vortex Spacing System (AVOSS) project (Hinton 1995; Perry et al. 1997). In 1997, the AVOSS setup at Memphis International Airport was relocated to Dallas-Fort Worth International Airport. The primary objective of this field deployment was to conduct a comprehensive test of various AVOSS procedures established at Memphis (Dasey et al. 1998; Joseph et al. 1999).

Following the successful AVOSS deployments, the Denver 2006 Out of Ground Effect (OGE) field experiment was conducted during the months of April to June. The primary objective of this data collection activity was to track OGE vortices in lateral transport further than previous campaigns. In order to accomplish this objective, the lidar placement (Figure 1) and configuration was such that the data collection focused on one direction of wake transport only (westerly winds).

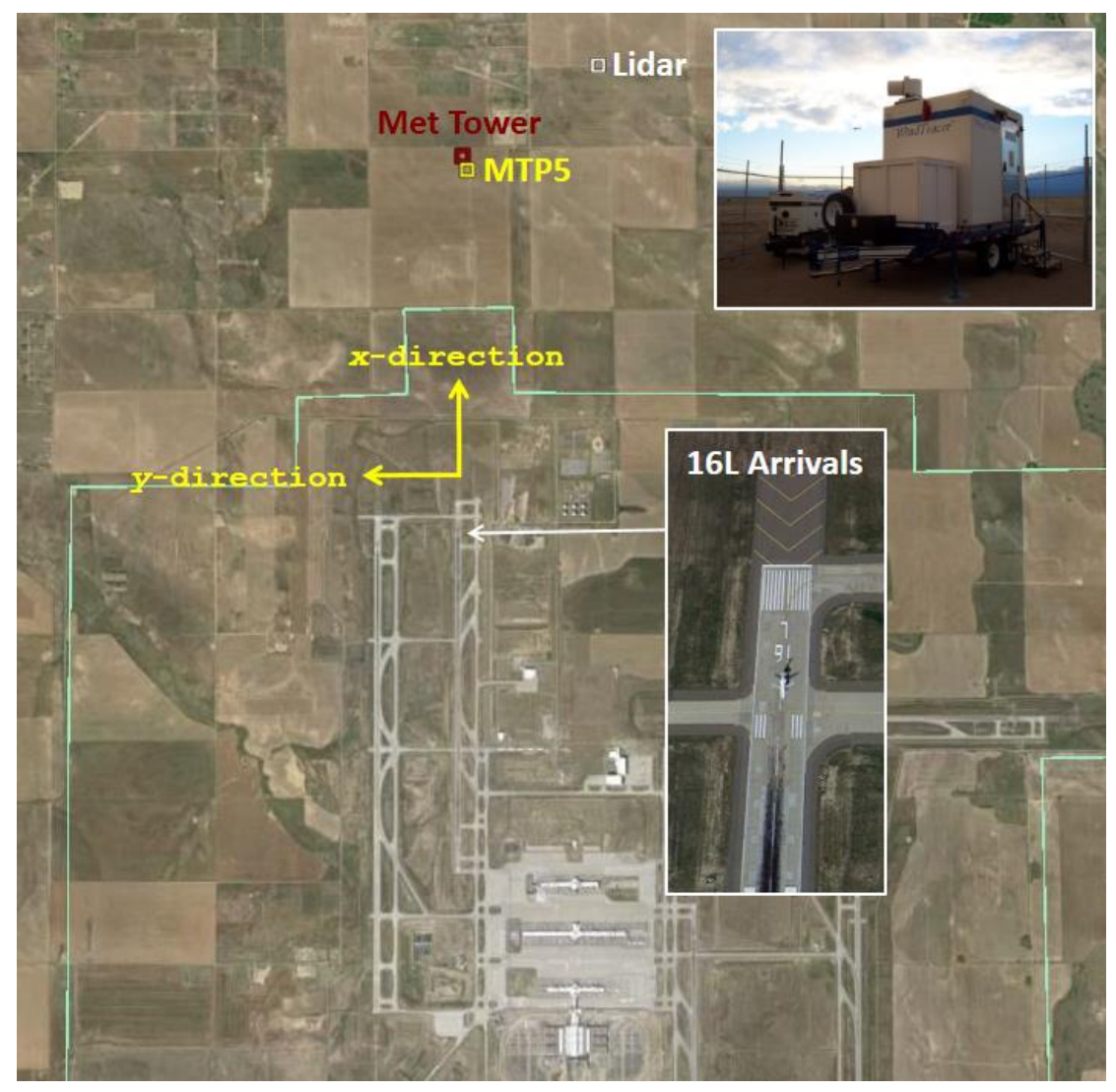

Figure 1. Google Earth image showing the placement of CTI Pulsed Lidar $\left(39^{\circ} 56^{\prime} 9.47^{\prime} \mathrm{N}, 104^{\circ} 40^{\prime} 12.46\right.$ ' $\left.\mathrm{W}\right)$,

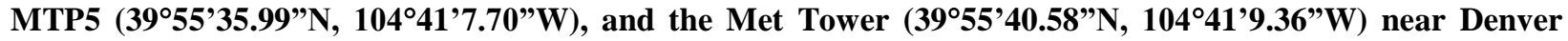
International Airport. Lidar was located at $x=14233.7 \mathrm{ft}, y=-4538.7 \mathrm{ft}$, and $z=-52.1 \mathrm{ft}$ from the $16 \mathrm{~L}$ extended centerline. 
The meteorological measurements needed for inputs into the fast-time wake vortex models were obtained using an MTP5 (temperature profile) and directly from the lidar observations (crosswind and EDR profiles). Additionally, measurements of total wind, turbulence and temperature measurements were also obtained near the surface at three heights on a $106 \mathrm{ft}$ tall meteorological tower. The altitudes of the sensors were at $7 \mathrm{~m}(23 \mathrm{ft}), 14.6 \mathrm{~m}(48 \mathrm{ft})$, and $32.3 \mathrm{~m}$ (103ft) AGL. Compared to the earlier AVOSS deployments which required extensive human interaction in the actual data collection, the Denver 2006 deployment was fully automated. The use of automation allowed data collection at different times of the day and in a much larger quantity. The Denver experiment was able to detect and track a large number of OGE wake vortex pairs, validate model assumptions regarding wake transport, and provide important data on the effect of wind shear and stratification on wake vortex transport.

In the following sections the Denver 2006 deployment and the processed dataset is described in detail. The fasttime wake vortex transport and decay prediction models were evaluated using the dataset and the model evaluation results are also presented.

\section{Denver Wake Vortex Field Experiment}

The data collected at Denver was processed to generate initial conditions for NASA's fast-time wake vortex models. The initial conditions for wake models include aircraft and meteorological data. The aircraft dependent parameters include the initial vortex descent velocity $\left(V_{0}\right)$, vortex pair separation distance $\left(b_{0}\right)$, the initial position (offset) of the vortex pair with respect to the runway centerline $\left(y_{0}\right)$ and the initial height of the vortices $\left(z_{0}\right)$. The atmospheric initial conditions include vertical profiles of potential temperature $(\theta)$, EDR $(\varepsilon)$ and crosswind. The atmospheric parameters that affect wake decay are atmospheric stratification, turbulence, and wind shear.

The original lidar data collected at Denver was reprocessed using the current version of the Lockheed Martin Coherent Technologies (LMCT) wake vortex algorithm and over 2,600 unique tracks were obtained. After matching the aircraft to known aircraft landings, approximately 2,100 aircraft landings were left in the dataset (primarily of "Large" category aircraft). This data was re-processed using WakeMod 2.5 algorithm. While the results for the LMCT legacy wake algorithm and WakeMod 2.5 are similar for Heavy aircraft, the WakeMod 2.5 algorithm gives improved circulation estimates for smaller aircraft compared to the Legacy algorithm (Jacob et al 2013). This difference is important for the Denver 2006 OGE data set which consists of mostly "Large" aircraft. The LMCT legacy position data is used in the WakeMod 2.5 algorithm, and WakeMod 2.5 does not produce or refine the LMCT legacy position estimate. The re-processing of data through WakeMod 2.5 did however result in another small reduction of total wake tracks, as some of the tracks did not pass WakeMod quality control factors. Finally, the amount of data available for use in the fast-time wake vortex models occurred due to gaps in the meteorological observations of either the stratification (809 landings) or EDR (2 landings). Figure 2 shows the distribution of aircraft types in the re-processed dataset.

In the following sections the WakeMod 2.5 re-processed subset of Denver 2006 wake data is presented along with accompanying meteorological data. The fast-time wake model evaluation was also conducted using this subset. The EDR data used for initializing the fast-time wake models was estimated from lidar measurements (Pruis and Delisi 2011). In the past data collections EDR have been estimated from sonic anemometers mounted on meteorological tower.

\section{A. Aircraft Data}

The aircraft data used by the fast-time wake prediction models include the initial position (offset) of the vortex pair with respect to the runway centerline $\left(y_{0}\right)$, the initial height of the vortices $\left(z_{0}\right)$, the initial vortex descent rate $\left(V_{0}\right)$ and the initial separation of vortices $\left(b_{0}\right)$. The initial position (offset) of the vortex pair with respect to the runway centerline $y_{0}$ was estimated using an average of the first few data points for each landing. The initial height of the vortices $z_{0}$ was estimated from backward extrapolation of the altitude trajectory in time. The initial separation distance between the vortices $b_{0}$ was estimated assuming an elliptical wing loading,

$$
b_{0}=\frac{\pi}{4} B
$$

where $B$ is the wingspan of the aircraft. The initial vortex descent rate was estimated from the aircraft weight, aircraft speed, air density, and the initial vortex separation $b_{0}$, 


$$
V_{0}=\frac{W}{2 \pi \rho U b_{0}^{2}}
$$

where $\rho$ is the air density - which was assumed to be $1.02 \mathrm{~kg} / \mathrm{m}^{3}$ for all the landings, $U$ is the airspeed, and $W$ is the landing weight of the aircraft, each estimated from reference tables.

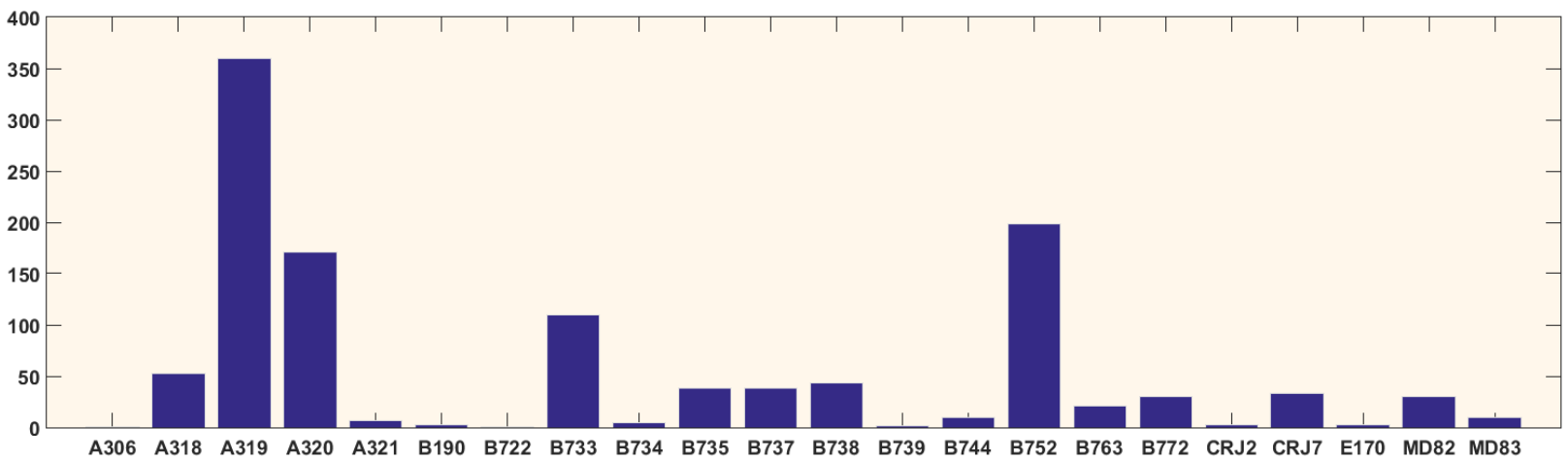

Figure 2. Aircraft Types in the Denver 2006 dataset re-processed using WakeMod 2.5 wake algorithm.

Figure 3 shows the distribution of initial conditions (initial vortex pair height, wingspan, aircraft weight, and airspeed) generated for fast-time wake models. The initial vortex height is estimated from measurements. The wingspan, aircraft weight and airspeed are obtained from a standard database.
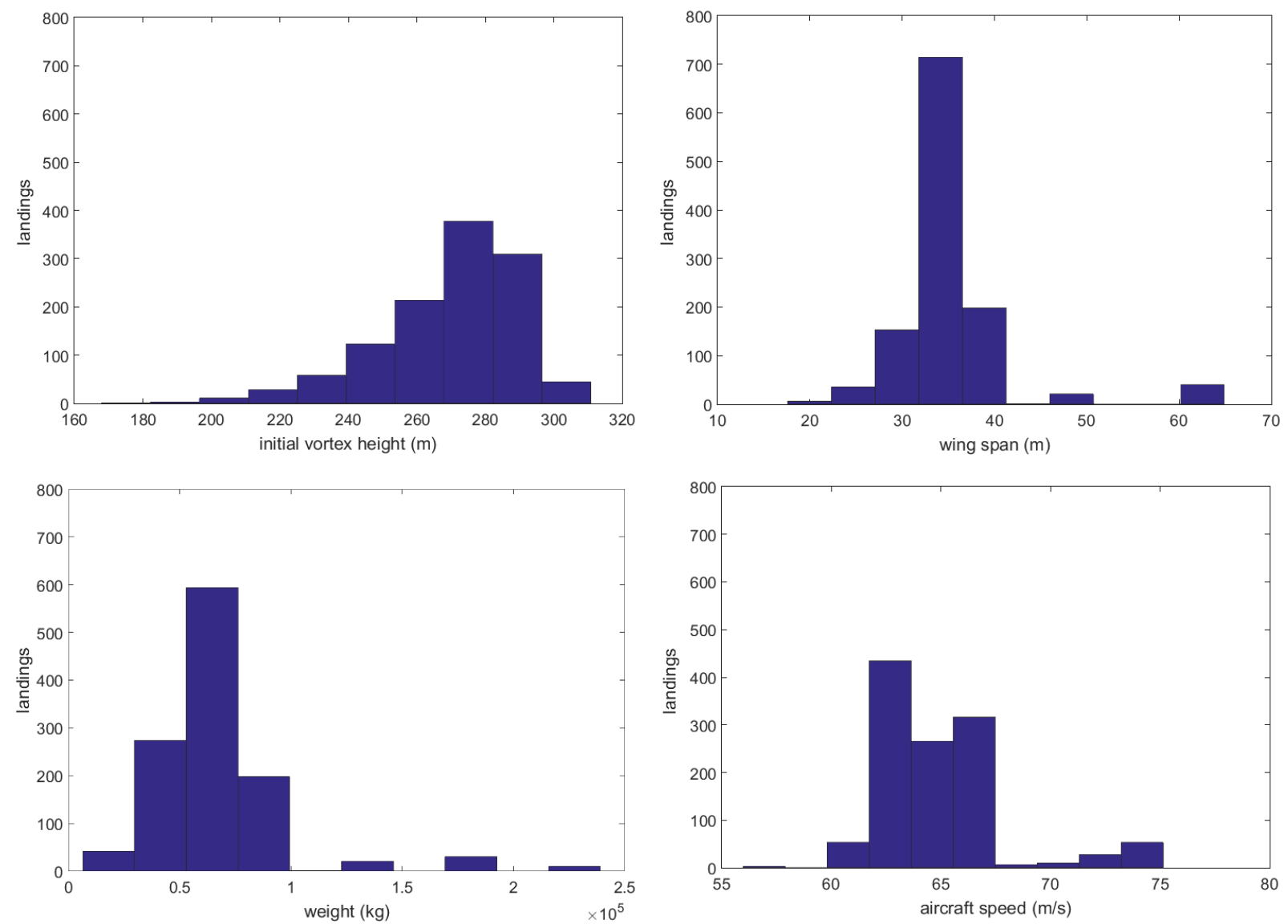

Figure 3. Histograms for aircraft related parameters are shown for all landings in the dataset. Initial vortex height (top left), wingspan (top right), aircraft weight (bottom left), and airspeed (bottom right). 


\section{B. Crosswinds and Headwinds}

Crosswind profiles were estimated using the average of two horizontal in-plane wind estimates from the lidar, where one profile was estimated just prior to the aircraft passage and one profile was estimated just after the end of the wake observations. Crosswind and headwind estimates are also available from the nearby ASOS sensor. Figure 4 shows the all crosswind data obtained from the lidar along with probability density fit using a normal distribution. The crosswind values range from $-13.4 \mathrm{~m} / \mathrm{s}$ to $10.4 \mathrm{~m} / \mathrm{s}$ with a mean of $-1.5 \mathrm{~m} / \mathrm{s}$.
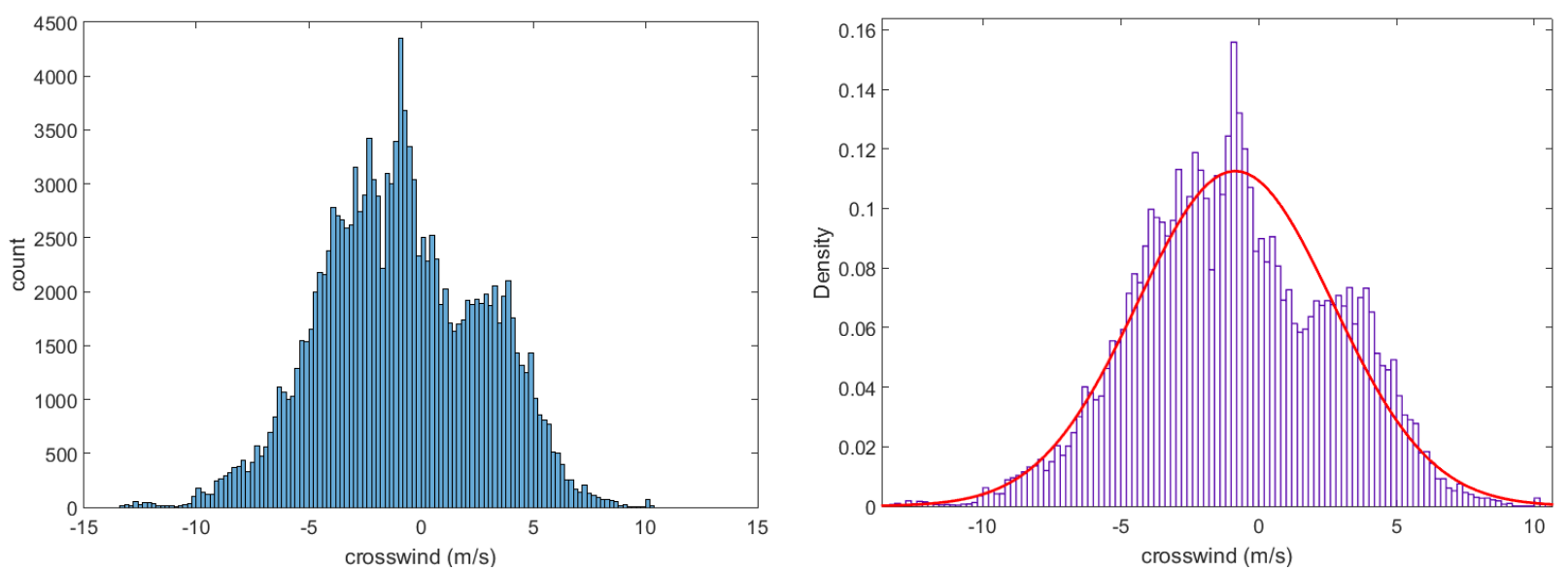

Figure 4. All crosswind data is shown in the left panel. The right panel shows the probability density fit for the data using a normal distribution $(\mu=-\mathbf{0 . 8 5 1}, \sigma=3.54$, and variance $=12.56)$.

\section{Eddy Dissipation Rate}

A vertical profile of EDR was generated by NASA obtained using the AVOSS Turbulence Profile Generator (ATPG) code which uses tower measurements of temperature and wind speed at two altitudes (Han et al. 2000; Joseph et al. 1999). Vertical profiles of EDR were also generated using the lidar data following the methodology described in Pruis and Delisi (2011). A benefit of using the lidar-based EDR estimates is that the observations are co-incident with the wake observations in time and space whereas the tower measurements require extrapolation of near-surface measurements and the accuracy of these extrapolation methods remains poorly quantified.

Figure 5 shows $\log 10(\varepsilon)$ values in the left panel and the probability density fit using a log-logistic distribution for the non-dimensional eddy dissipation rates. EDR estimates from the pulsed lidar are plotted in the figure.
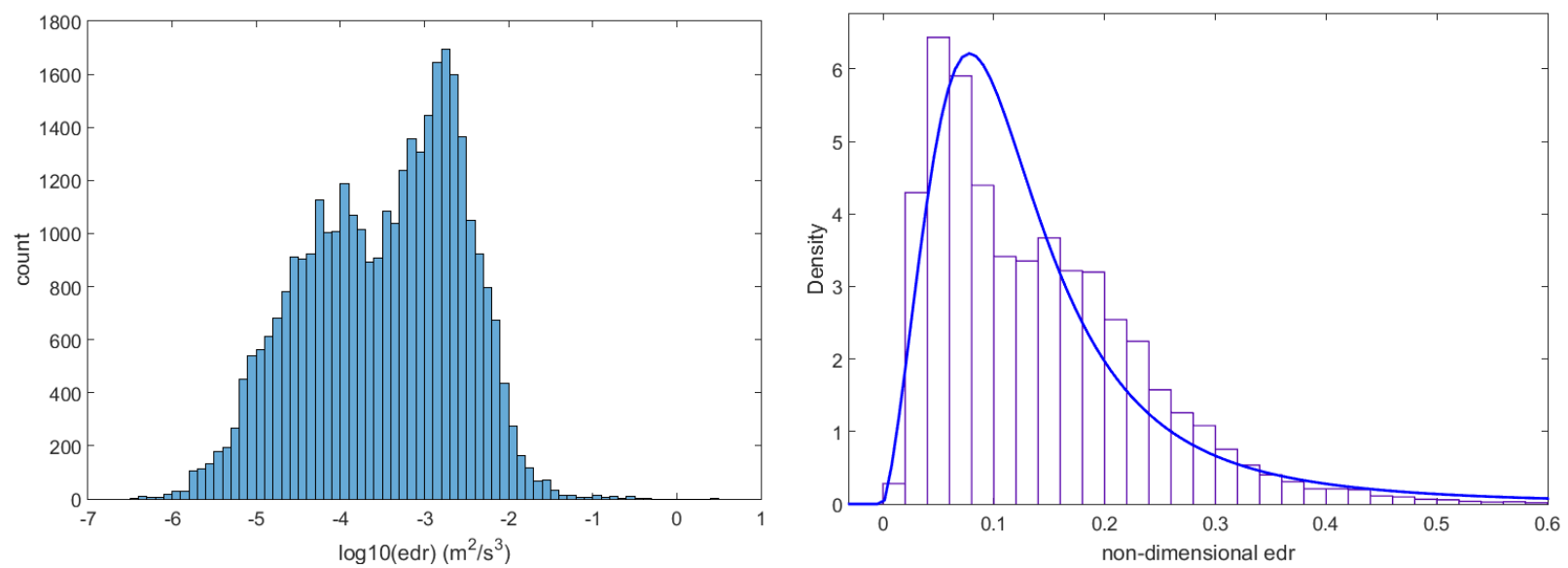

5

American Institute of Aeronautics and Astronautics 
Figure 5. Eddy dissipation rates data is shown in the left panel. The right panel shows the probability density function fit for $\varepsilon^{*}$ using a $\log$-logistic distribution $(\mu=0.156, \sigma=0.423$, and variance $=0.05)$.

\section{Stratification}

The MTP5 estimated air temperature up to a height of $600 \mathrm{~m}$ with one estimate every $50 \mathrm{~m}$ above the sensor. These temperature estimates were converted to potential temperature using a dry adiabatic lapse rate $(0.00976 \mathrm{~K} / \mathrm{m})$.

Figure 6 (left panel) shows the potential temperature gradient calculated from each profile. The current versions of fast-time models assume a neutral stratification in case of negative potential temperature gradient. In Figure 6 (right panel), all negative gradients were set to zero before calculating the non-dimensional Brunt-Väisälä frequency.
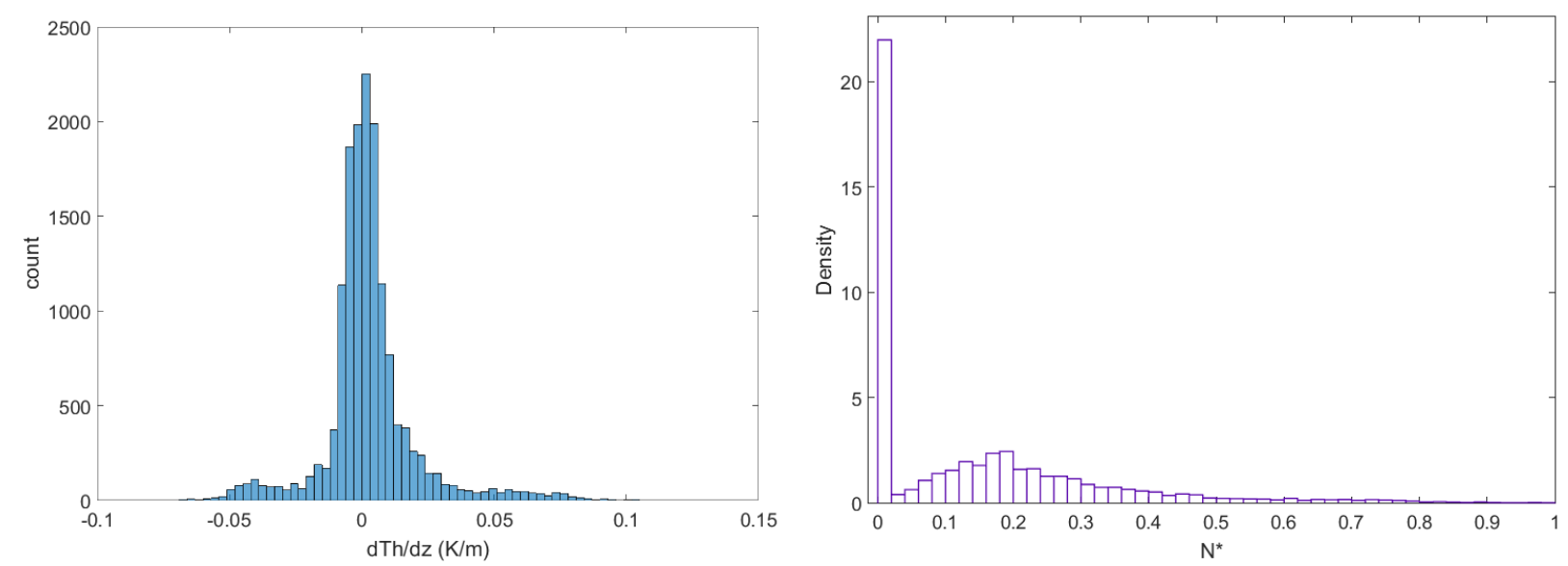

Figure 6. All potential temperature gradient data is shown in the left panel. The right panel shows the probability density function for non-dimensional Brunt-Väisälä frequency $\left(N^{*}\right)$.

\section{E. Lidar Data}

Lidar data was collected using an LMCT Windtracer®. The experimental design and setup were optimized for measuring long lateral transport and descent of wake vortices. Wake trajectories were estimated using the Lockheed Martin legacy code. Previous studies indicate that the mean biases for position in the lateral direction are 4 to $7 \mathrm{~m}$ and 1 to $3 \mathrm{~m}$ in the vertical direction for typical out of ground effect (OGE) data collection geometry and processing parameters (Lai et al 2010). Wake strength was estimated using WakeMod 2.5 (Jacob et al 2013). The new wake processing algorithm appears to give improved circulation estimates for smaller aircraft than is obtained with the Legacy algorithm. This difference is important for the Denver 2006 OGE data set which consists of mostly of "Large" aircraft.

\section{Evaluation of Fast-Time Wake Transport and Decay Models}

The re-processed Denver 2006 lidar tracks using WakeMod 2.5 algorithm were used to evaluate the current versions of NASA's fast-time wake models. The EDR used for model initialization is usually obtained from sonic anemometers. For this evaluation, the models were initialized using EDR estimated from the pulsed lidar. A brief description of the models and the results of model evaluation are presented in this section.

Fast-time wake models are empirical or semi-empirical algorithms used for real-time predictions of wake transport and decay based on aircraft parameters and ambient weather conditions (Ahmad et al. 2014). The first fast-time wake transport and decay model was developed by Greene (1986). NASA's fast-time models include: AVOSS (Aircraft Vortex Spacing System) Prediction Algorithm (APA), and TASS (Terminal Area Simulation System) Derived Algorithms for Wake Prediction (TDP). The APA model (Robins and Delisi 2002) incorporates Sarpkaya's model (Sarpkaya 2000; Sarpkaya et al. 2001) for out-of-ground-effect vortex decay and descent, and follows an approach similar to Corjon and Poinsot (1996) for in-ground-effect vortex transport. In the TDP model (Proctor et al. 2006; Proctor and Hamilton 2009) the Sarpkaya component is replaced with algorithms developed from parametric studies using large eddy simulation (LES) model. The current version of the TDP model includes the effect of the crosswind shear gradient on transport. 
The current versions of the fast-time models were evaluated using the lidar observations from the Denver field experiment (Table 1). Proxy crosswinds (Pruis et al. 2011) were not used in this analysis. The accuracy of predictions for the models was quantified in terms of root mean square error $\left(\right.$ Error $\left._{\mathrm{rms}}\right)$, mean absolute error $\left(\right.$ Error $\left._{\mathrm{mae}}\right)$, and Bias:

$$
\text { Error }_{\text {rms }}=\sqrt{\frac{1}{n} \sum_{i=1}^{n}\left(x_{i}^{a p a}-x_{i}^{o b s}\right)^{2}} ; \quad \text { Error }_{\text {mae }}=\frac{1}{n} \sum_{i=1}^{n}\left|x_{i}^{a p a}-x_{i}^{o b s}\right| ; \quad \text { Bias }=\frac{1}{n} \sum_{i=1}^{n}\left(x_{i}^{a p a}-x_{i}^{o b s}\right)
$$

The evaluation statistics using the Memphis 1995 dataset are given in Table 2 as a reference. There are several differences between the Memphis 1995 and Denver 2006 datasets, which include - different sensors and processing methodologies for generating meteorological initial conditions and the use of different wake sensors. The Memphis 1995 dataset was also used in the APA model development (TDP is based on large eddy simulations). The Denver model evaluation shows good consistency in model predictions of circulation decay, lateral transport and vortex descent. The APA models were tuned using Memphis 1995 data but exhibit similar behavior when evaluated using the much larger Denver dataset (Denver dataset has five times the OGE cases compared to the Memphis dataset). The low bias in model prediction of lateral transport in the Denver evaluation also indicates good wind observations.

Table 1: Fast-Time Models Evaluation using Denver 2006 Data - (1170 OGE Cases)

\begin{tabular}{ccccccccccc}
\hline \multirow{2}{*}{ Model } & \multicolumn{2}{c}{$\begin{array}{c}\text { Circulation } \\
\left.\text { (normalized by } \Gamma_{\mathbf{0}}\right)\end{array}$} & \multicolumn{2}{c}{$\begin{array}{c}\text { Lateral Transport } \\
\left(\text { normalized by } \boldsymbol{b}_{\mathbf{0}}\right)\end{array}$} & \multicolumn{3}{c}{$\begin{array}{c}\text { Altitude } \\
\left(\text { normalized by } \boldsymbol{b}_{\mathbf{0}}\right)\end{array}$} \\
\cline { 2 - 10 } & rmse & mae & bias & rmse & mae & bias & rmse & mae & bias \\
\hline TDP2.1 & 0.296 & 0.275 & 0.211 & 0.814 & 0.754 & -0.015 & 0.652 & 0.606 & -0.389 \\
\hline APA3.4 & 0.240 & 0.217 & 0.136 & 0.815 & 0.755 & -0.003 & 0.619 & 0.574 & -0.305 \\
\hline APA3.2 & 0.231 & 0.205 & 0.072 & 0.820 & 0.755 & 0.006 & 0.618 & 0.572 & 0.618 \\
\hline
\end{tabular}

Table 2: Fast-Time Models Evaluation using Memphis 1995 Data - (219 OGE Cases)

\begin{tabular}{ccccccccccc}
\hline \multirow{2}{*}{ Model } & \multicolumn{2}{c}{$\begin{array}{c}\text { Circulation } \\
\left(\text { (normalized by } \Gamma_{\mathbf{0}}\right)\end{array}$} & \multicolumn{2}{c}{$\begin{array}{c}\text { Lateral Transport } \\
\left(\text { normalized by } \boldsymbol{b}_{\mathbf{0}}\right)\end{array}$} & \multicolumn{3}{c}{$\begin{array}{c}\text { Altitude } \\
\left(\text { normalized by } \boldsymbol{b}_{\mathbf{0}}\right)\end{array}$} \\
\cline { 2 - 10 } & rmse & mae & bias & rmse & mae & bias & rmse & mae & bias \\
\hline TDP2.1 & 0.254 & 0.218 & 0.037 & 1.061 & 0.871 & 0.166 & 0.588 & 0.500 & 0.065 \\
\hline APA3.4 & 0.232 & 0.200 & -0.042 & 1.021 & 0.839 & 0.181 & 0.608 & 0.517 & 0.156 \\
\hline APA3.2 & 0.242 & 0.210 & -0.115 & 1.045 & 0.856 & 0.193 & 0.609 & 0.520 & 0.212 \\
\hline
\end{tabular}

\section{Summary}

The primary objective of this paper was to give an overview of NASA's Denver 2006 field deployment. A summary of the Denver 2006 dataset and an evaluation of NASA's fast-time wake model using the wake observations were presented. The newly re-processed lidar tracks using WakeMod 2.5 algorithm were used to evaluate the current 
versions of NASA's fast-time models. The errors in model circulation predictions had a mean root mean square error on the order of $0.2 \Gamma_{0}$ to $0.3 \Gamma_{0}$ ( $\Gamma_{0}$ is the initial wake circulation), the vertical transport errors were on the order of $0.5 b_{0}$ and the lateral transport errors were on the order of $b_{0}$. These values are consistent with previous evaluations of the models using other wake datasets.

\section{Acknowledgments}

This work is sponsored under NASA's Aviation Operations and Safety Program. Numerous people were involved in various stages of the field program planning, setup/installation, and data processing including Kevin Clark, Steve Mackey, Melanie Soares, Amir Tabrizi, Frank Wang, and Hadi Wassaf. Others were involved in site survey, sensor setup, site acceptance and various iterations of post processing, including David Hamilton, Keith Barr, Steve Hannon, Leo Jacobs, and Ken Underwood. Their assistance is gratefully acknowledged. Many thanks to Dr. Frank Wang of the Volpe National Transportation Systems Center for providing documentation on the field experiment.

\section{References}

Ahmad, NN, R. VanValkenburg, MJ Pruis, "NASA AVOSS Fast-Time Wake Prediction Models: User's Guide,” National Aeronautics and Space Administration, NASA/TM-2014-218152.

Campbell, SD, et al., "Wake Vortex Field Measurement Program at Memphis, TN Data Guide", Lincoln Laboratory, Massachusetts Institute of Technology. Project Report NASA/L-2. 1997.

Chow, J, G Zilliac, and P Bradshaw, “Turbulence Measurements in the Near Field of a Wingtip Vortex", NASA-TM 110418, 1997.

Corjon, A, and T Poinsot, "Vortex Model to Define Safe Aircraft Separation Distances," Journal of Aircraft, Vol. 33, No.3, May-June 1996, pp. 547-553.

Dasey, TJ, et al., “Aircraft Vortex Spacing System (AVOSS). Initial 1997 System Deployment at Dallas/Ft. Worth (DFW) Airport”, Lincoln Laboratory, Massachusetts Institute of Technology. Project Report NASA/L-3. 1998.

Dougherty, RP, FY Wang, ER Booth, ME Watts, N Fenichel, RE D’Errico, “Aircraft Wake Vortex Measurements at Denver International Airport," American Institute of Aeronautics and Astronautics, AIAA-2004-2880.

Greene, GC, “An Approximate Model of Vortex Decay in the Atmosphere”, Journal of Aircraft, Vol. 23, 1986, pp. $566-573$.

Hinton, DA, “Aircraft Vortex Spacing System (AVOSS) Conceptual Design”, NASA Technical Memorandum NASA-TM110184, 1995.

Han, J, SP Arya, S Shen, Y Lin, "An Estimation of Turbulent Kinetic Energy and Energy Dissipation Rate Based on Atmospheric Boundary Layer Similarity Theory”, NASA Contractor Report NASA-CR-2000-210298. 2000.

Jacob, D, D Lai, and D Delisi, "Development of an Improved Pulsed Lidar Circulation Estimation Algorithm and Performance Results for Denver OGE Data", American Institute of Aeronautics and Astronautics, 10.2514/6.2013-509.

Joseph, R, T Dasey, R Heinrichs, "Vortex and Meteorological Measurements at Dallas/Ft. Worth Airport," American Institute of Aeronautics and Astronautics, AIAA-1999-0760.

Lai, DY, Jacob, D, and Delisi, DP, “Assessment of Pulsed Lidar Measurements of Aircraft Wake Vortex Position Using a Lidar Simulator,” American Institute of Aeronautics and Astronautics, AIAA 2010-7988, 2010.

Perry, RB, DA Hinton, and RA Stuever, “NASA Wake Vortex Research for Aircraft Spacing,” AIAA Paper 1997-0057. 1987.

Proctor, FH, “The Terminal Area Simulation System / Volume 1: Theoretical Formulation”, NASA Technical Report 4046.

Proctor, FH, DW Hamilton, GF Switzer, “TASS Driven Algorithms for Wake Prediction," American Institute of Aeronautics and Astronautics, AIAA-2006-1073.

Proctor, FH, "Evaluation of Fast-Time Wake Vortex Prediction Models," American Institute of Aeronautics and Astronautics, AIAA-2009-0344.

Pruis, MJ, DP Delisi, NN Ahmad, "Comparisons of Crosswind Velocity Profile Estimates Used in Fast-Time Wake Vortex Prediction Models," AIAA Paper 2011-1002.

Pruis, MJ, DP Delisi, NN Ahmad, and FH Proctor, “Atmospheric Turbulence Estimates from a Pulsed Doppler Lidar", American Institute of Aeronautics and Astronautics, 10.2514/6.2013-512, 2013.

Robins, RE, and DP Delisi, "NWRA AVOSS Wake Vortex Prediction Algorithm Version 3.1.1,” NASA CR 2002-211746. 
Sarpkaya, T, "New Model for Vortex Decay in the Atmosphere," Journal of Aircraft, Vol. 37, 2000, pp. 53-61.

Sarpkaya, T, RE Robins, and DP Delisi, "Wake-Vortex Eddy-Dissipation Model Predictions Compared with Observations," Journal of Aircraft, Vol. 38, 2001, pp. 687- 692.

Vicroy, DD, PM Vijgen, HM Reimer, JL Gallegos, PR Spalart, "Recent NASA Wake-Vortex Flight Tests, Flow Physics Database and Wake-Development Analysis,” AIAA Paper 1998-5592.

Wang, F, "Quick Reference for the DEN06 OGE Lidar Data”, PowerPoint Presentation (Personal communication). 